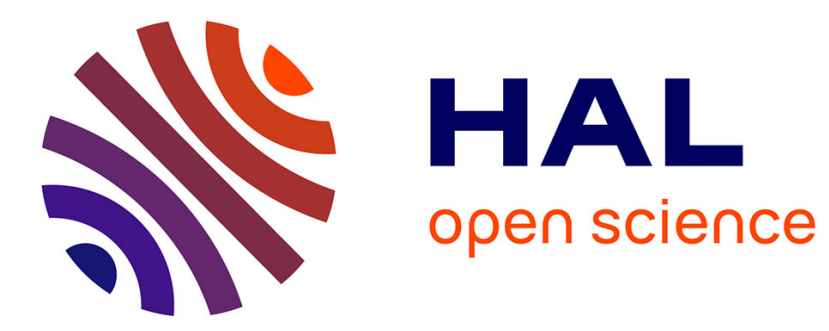

\title{
Uncertainty theory as a basis for belief reliability
}

Zhiguo Zeng, Rui Kang, Meilin Wen, Enrico Zio

\section{To cite this version:}

Zhiguo Zeng, Rui Kang, Meilin Wen, Enrico Zio. Uncertainty theory as a basis for belief reliability. Information Sciences, 2018, 429, pp.26 - 36. 10.1016/j.ins.2017.10.050 . hal-01632274

\section{HAL Id: hal-01632274 https://hal.science/hal-01632274}

Submitted on 9 Nov 2017

HAL is a multi-disciplinary open access archive for the deposit and dissemination of scientific research documents, whether they are published or not. The documents may come from teaching and research institutions in France or abroad, or from public or private research centers.
L'archive ouverte pluridisciplinaire HAL, est destinée au dépôt et à la diffusion de documents scientifiques de niveau recherche, publiés ou non, émanant des établissements d'enseignement et de recherche français ou étrangers, des laboratoires publics ou privés. 


\title{
Uncertainty theory as a basis for belief reliability
}

\author{
Zhiguo Zeng, PhD ${ }^{\mathrm{a}}$, Rui Kang, Professor ${ }^{\mathrm{b}, *}$, Meilin Wen, Assistant professor ${ }^{\mathrm{b}}$, \\ Enrico Zio, Professor ${ }^{\mathrm{a}, \mathrm{c}}$ \\ ${ }^{a}$ Chair System Science and the Energy Challenge, Fondation Electricité de France (EDF), \\ CentraleSupélec, Université Paris-Saclay, Grande Voie des Vignes, 92290 \\ Chatenay-Malabry, France \\ ${ }^{b}$ School of Reliability and Systems Engineering, Beihang University \\ ${ }^{c}$ Energy Department, Politecnico di Milano
}

\begin{abstract}
Belief reliability is a newly developed, model-based reliability metric which considers both what we know (expressed as reliability models) and what we don't know (expressed as epistemic uncertainty in the reliability models) about the reliability. In this paper, we show that due to the explicit representation of epistemic uncertainty, belief reliability should not be regarded as a probability measure; rather, it should be treated as an uncertain measure in uncertainty theory. A minimal cut set-based method is developed to calculate the belief reliability of coherent systems. A numerical algorithm is, then, presented for belief reliability analysis based on fault tree models. The results of application show that the developed methods require less computations than the structure function-based method of classical reliability theory.
\end{abstract}

Keywords: Reliability, epistemic uncertainty, uncertainty theory, belief reliability, fault tree,

\section{1. Introduction}

2 Modern reliability engineering is increasingly looking at the model-based 3 methods (cf. physics-of-failure (PoF) methods [5], structural reliability methods

\footnotetext{
${ }^{*}$ Corresponding author

Email addresses: zhiguo.zeng@centralesupelec.fr (Zhiguo Zeng, PhD), kangrui@buaa.edu.cn (Rui Kang, Professor), wenmeilin@buaa.edu.cn (Meilin Wen, Assistant professor), enrico.zio@ecp.fr (Enrico Zio, Professor)

Preprint submitted to Information Sciences

March 13, 2017
} 
1 [6], etc.), where reliability is predicted exploiting deterministic failure behavior

2 models whose parameter variations are assumed to be the only source of un-

3 certainty [37]. In practice, however, apart from the random variations in the

4 model parameters (often referred to as aleatory uncertainty [1]), the predicted

5 reliability is also subject to the influence of epistemic uncertainty due to incom-

6 plete knowledge on the degradation and failure processes [20]: for example, the

7 developed failure behavior model might not be able to accurately describe the

8 actual failure process; besides, the precise values of the model parameters might

9 not be accurately estimated [2, 4], etc. In most existing model-based reliability

10 assessment methods, however, the effect of epistemic uncertainty has not been

11 considered.

12 Recently, a new metric of reliability, the belief reliability, has been defined to

13 explicitly account for epistemic uncertainty in model-based reliability analysis

14 and assessment $[10,35,37]$. The new reliability metric integrates the contri-

15 butions of design margin, aleotory uncertainty and epistemic uncertainty and

16 provides a more comprehensive and systematic description of reliability. Zeng

17 et al. [37] presented a framework to evaluate the belief reliability where epis-

18 temic uncertainty is quantified by the effectiveness of the engineering analysis

19 and assessment activities that contribute to the state of knowledge on the fail-

20 ure causes and processes. Belief reliability has been applied successfully on the

21 reliability evaluation of hydraulic servo-actuators [35, 37], DC regulated pow-

22 er supplies [10] and printed circuit boards [17], all of which are subject to the

23 influence of epistemic uncertainty.

24 Currently, the belief reliability of a component or a system can only be e25 valuated from its definition (i.e., based on design margin, aleotory uncertainty 26 and epistemic uncertainty) [35]. In practice, we often need to calculate the 27 belief reliability of a system based on the structure of the system and the be28 lief reliabilities of its components (referred to as system reliability analysis in 29 conventional reliability theories [31]). To address this problem, a mathematical

30 theory should be determined as the mathematical foundation of belief reliability, 31 based on which the system belief reliability analysis method can be developed. 
1 In literature, various mathematical theories have been used to describe epistemic

2 uncertainty, e.g., probability theory (subjective interpretation [7]), evidence the3 ory [29], possibility theory [8] and uncertainty theory [26], etc. Kang et al. [18]

4 reviewed the theories and concluded that among them, uncertainty theory is

5 the most suitable one for modeling belief reliability since it satisfies the Duality

6 Axiom and adopts minimum operation as the Product Axiom, which are two

7 essential requirements for a mathematical theory qualified to describe reliability

8 under the influence of epistemic uncertainty. If either requirement is violated,

9 misleading results might be reached when belief reliability is applied in practical

10 applications (see Section 3.2 for a detailed discussion).

11 Uncertainty theory, proposed by Liu in 2007 [21] and refined by Liu in 2010

12 [24], is a branch of axiomatic mathematic founded on four axioms, the Normal-

13 ity, Duality, Subadditivity and Product Axiom. Currently, uncertainty theory

14 has been widely applied in various fields, including portfolio selection [38], net-

15 work science [14], option pricing [16], graph theory [13], transportation [32],

16 supply chain [15], etc. The research of reliability in uncertainty theory started

17 from [23], where Liu defined the reliability index and showed how to calculate

18 the system reliability index from the system structure functions. In [27], the

19 reliability indexes for redundant systems were calculated for the case in which

20 the lifetimes of the components are uncertain variables. Zeng et al. [36] defined

21 time-static and time-variant reliability in the context of uncertainty theory and

22 developed calculation methods for the reliability indexes. Wen and Kang [30]

23 developed an approach to calculate the reliability index when both uncertain

24 variables and random variables are considered. Gao and Yao [12] investigated

25 the importance index in the context of uncertainty theory. Age replacement

26 and block replacement policies were also investigated with lifetimes described

27 as uncertain variables [19, 39, 40].

28 Most existing system reliability analysis methods in uncertainty theory are 29 based on structure functions (e.g., see [23] and [30]). Since they require enumer-

30 ating all the possible combination of system states, the computational efficiency

31 of the structure function-based methods are often unsatisfactory, especially for 
1 large and complex systems. In a previous study, minimal cut sets have been used

2 to alleviate the computational burdens of the structure function-based method-

3 s [36]. However, the method developed in [36] requires independence among

4 the minimal cut sets, which is a strong condition and restricts its application.

5 In this paper, we show that the restriction is unnecessary and develop a min-

6 imal cut set-based method to calculate the belief reliability for a system with

7 independent components.

8 The rest of this paper is organized as follows. Section 2 reviews the definition

9 of belief reliability. In Section 3, we justify the choice of uncertainty theory as

10 the mathematical foundation of belief reliability and give the definition of belief

11 reliability in the context of uncertainty theory. Then, a system belief reliability

12 analysis method is developed based on minimal cut sets in Section 4. In Section

135 , a numerical algorithm is presented for belief reliability analysis based on fault

14 tree models. The paper is concluded in Section 6 with discussions on possible

15 future research directions.

\section{2. Definition of belief reliability}

17 In traditional model-based reliability methods, it is assumed that the failure

18 behavior of a component or system is characterized by its performance margin

$19 m$, which is modeled by:

$$
m=g_{m}(\mathbf{x}),
$$

20 where $m \leq 0$ indicates that the component or system fails and $m>0$ indicates 21 normal functioning; $g_{m}(\cdot)$ is developed by modeling the failure process [34].

22 Given the probability density functions of the input variables $\mathbf{x}$, denoted by

$23 f_{X}(\mathbf{x})$, the reliability index can be calculated as

$$
R_{p}=\operatorname{Pr}\left(g_{m}(\mathbf{x})>0\right)=\int \cdots \int_{g_{m}(\mathbf{x})>0} f_{X}(\mathbf{x}) \mathrm{d} \mathbf{x} .
$$

24 To differentiate it from belief reliability, the reliability index in (2) is referred

25 to as probabilistic reliability in this paper. 
1 In the model-based reliability methods, a fundamental assumption is that,

2 the reliability model is correct and accurate, so that all the uncertainty comes

3 from the random variations in $\mathrm{x}$ (aleatory uncertainty). The validity of such an

4 assumption heavily depends on the state-of-knowledge we have on the failure

5 process. In a lot of practical applications, however, due to the limitation of the

6 knowledge, the models in (1) and (2) might not be able to accurately capture

7 the actual failure process. Besides, the precise values of the model parameters

8 might not be accurately known to us. Therefore, the predicted reliability in-

9 dex is subject to an additional source of uncertainty, which arises from lack of

10 knowledge and is referred to as epistemic uncertainty [41].

11 Belief reliability was proposed as a metric of reliability that explicitly ac12 counts for epistemic uncertainty in reliability analysis and assessment $[10,35$,

13 37]. Note that in (1) and (2), the probabilistic reliability $R_{p}$ can be viewed

14 as determined by deterministic designs and aleatory uncertainty in the design

15 parameters. Deterministic designs are quantified by design margin $m_{d}$ :

$$
m_{d}=g_{m}\left(\mathbf{x}_{N}\right)
$$

16 where $\mathbf{x}_{N}$ is the nominal values of the parameters. Aleatory uncertainty is 17 measured by $F_{a}$, the factor of aleatory uncertainty, which is defined by:

$$
F_{a}=\frac{m_{d}}{Z_{R_{p}}}
$$

18 where $R_{p}$ is given by (1) and (2); $Z_{\alpha}$ is the value of the inverse cumulative 19 distribution function of a standard normal distribution evaluated at $\alpha$. Let us 20 define equivalent performance margin $M_{E}$ as:

$$
M_{E}=m_{d}+\epsilon_{a},
$$

21 where $m_{d}$ is the design margin in $(3)$ and $\epsilon_{a} \sim \operatorname{Normal}\left(0, F_{a}^{2}\right)$ quantifies the 22 effect of aleatory uncertainty. It is easy to verify that $M_{E} \sim \operatorname{Normal}\left(m_{d}, F_{a}^{2}\right)$

23 and the probabilistic reliability $R_{p}$ can be calculated as the probability that $24 M_{E}>0$, as shown in Figure 1 (a). 
1 In belief reliability, epistemic uncertainty is described by introducing a factor

2 of epistemic uncertainty, denoted by $F_{e}$, whose value is related to the state-of-

3 knowledge of the failure processes and is measured based on the effectiveness

4 of the engineering analysis and assessment activities for component and system

5 reliability performance characterization [10, 37]. An adjustment factor $\epsilon_{e} \sim$

$6 \operatorname{Normal}\left(0, F_{e}^{2}\right)$ is introduced to quantify the effect of epistemic uncertainty on

7 the equivalent performance margin:

$$
M_{E}=m_{d}+\epsilon_{a}+\epsilon_{e} .
$$

8 Equation (6) indicates that epistemic uncertainty introduces additional disper-

9 sion to the aleatory distribution of the equivalent performance margin, as shown

10 in Figure 1 (b). Considering (6) and the normality assumption on $\epsilon_{a}$ and $\epsilon_{e}$,

11 belief reliability is defined as:

12 Definition 1 (Belief reliability [37]). The reliability metric

$$
R_{B}=\Phi_{N}\left(\frac{m_{d}}{\sqrt{F_{a}^{2}+F_{e}^{2}}}\right)
$$

13 is defined as belief reliability, where $\Phi_{N}(\cdot)$ is the cumulative distribution function 14 of a standard normal random variable.

15 It can be shown from (7) that as $F_{e} \rightarrow 0, R_{B} \rightarrow R_{p}$, where $R_{p}$ denotes 16 the conventional model-based reliability metric calculated under the same con17 ditions. This is natural, since $F_{e} \rightarrow 0$ indicates that there is no epistemic 18 uncertainty and, therefore, the failure behavior can be accurately determined 19 by the reliability models in (1) and (2).

20 In practical application, we always have $m_{d}>0$ and $F_{e} \geq 0$ [37]. It is easy 21 to verify from (7) that

$$
R_{B} \leq R_{p}
$$

22 which shows that using belief reliability yields a more conservative evaluation 23 result than using the probability-based reliability metric. The reason is that 24 belief reliability considers the effect of insufficient knowledge on the estimated 
1 reliability, while the probability-based reliability metric implicitly assumes that

2 knowledge is complete. It is the additional uncertainty caused by the insufficient

3 knowledge that reduces our confidence on the reliability estimation.

4 3. Uncertainty theory as the mathematical foundation of belief reli5 ability

6 In this section, we discuss the mathematical foundations of belief reliabili-

7 ty and show that the new reliability metric should be modeled by uncertainty

8 theory. Uncertainty theory is reviewed in subsection 3.1. In subsection 3.2, we

9 explain the reasons to choose uncertainty theory as the mathematical founda-

10 tion, and then define belief reliability as an uncertain measure.

\section{3.1. Preliminaries of Uncertainty Theory}

The first important concept in uncertainty theory is that of an event. Let $\Gamma$

13 be a nonempty set, and $\mathcal{L}$ a $\sigma$-algebra over $\Gamma$. Each element $\Lambda$ in $\mathcal{L}$ is called an 14 event.

15 In uncertainty theory, the belief degree of an event is measured by its un16 certain measure. An uncertain measure is a set function $\mathcal{M}$ from $\mathcal{L}$ to $[0,1]$ 17 satisfying the following three axioms [21]:

18 Axiom 1 (Normality Axiom [21]). $\mathcal{M}\{\Gamma\}=1$ for the univeral set $\Gamma$.

19 Axiom 2 (Duality Axiom [21]). $\mathcal{M}\{\Lambda\}+\mathcal{M}\left\{\Lambda^{c}\right\}=1$ for any event $\Lambda$.

20 Axiom 3 (Subadditivity Axiom [21]). For every countable sequence of events $21 \Lambda_{1}, \Lambda_{2}, \cdots$,

$$
\mathcal{M}\left\{\bigcup_{i=1}^{\infty} \Lambda_{i}\right\} \leq \sum_{i=1}^{\infty} \mathcal{M}\left\{\Lambda_{i}\right\} .
$$

22 The triplet $(\Gamma, \mathcal{L}, \mathcal{M})$ is called an uncertainty space [21]. A product uncertain 23 measure was defined by Liu [22] in order to obtain an uncertain measure of a 24 compound event, thus producing the fourth axiom of uncertainty theory: 
1 Axiom 4 (Product Axiom [22]). Let $\left(\Gamma_{k}, \mathcal{L}_{k}, \mathcal{M}_{k}\right)$ be uncertainty spaces for $k=$

$21,2, \cdots$ The product uncertain measure $\mathcal{M}$ is an uncertain measure satisfying

$$
\mathcal{M}\left\{\prod_{k=1}^{\infty} \Lambda_{k}\right\}=\bigwedge_{k=1}^{\infty} \mathcal{M}_{k}\left\{\Lambda_{k}\right\}
$$

3 where $\Lambda_{k}$ are arbitrarily chosen events from $\mathcal{L}_{k}$ for $k=1,2, \cdots$, respectively.

4 An uncertain variable is a measurable function $\xi$ from an uncertainty space

$5(\Gamma, \mathcal{L}, \mathcal{M})$ to the set of real numbers, i.e. , for any Borel set $B$ of real numbers,

6 the set $\{\xi \in B\}=\{\gamma \in \Gamma \mid \xi(\gamma) \in B\}$ is an event [21].

7 In practice, an uncertain variable is described by the uncertainty distribution

8 [21], defined by

$$
\Phi(x)=\mathcal{M}\{\xi \leq x\}, \forall x \in \Re
$$

9 An uncertainty distribution is said to be regular if its inverse function $\Phi^{-1}(\cdot)$

10 exists and is unique for each $\alpha \in(0,1)$ [24].

11 The uncertain variables $\xi_{1}, \xi_{2}, \ldots, \xi_{m}$ are said to be independent if

$$
\mathcal{M}\left\{\bigcap_{i=1}^{m}\left(\xi_{i} \in B_{i}\right)\right\}=\bigwedge_{i=1}^{m} \mathcal{M}\left\{\xi_{i} \in B_{i}\right\}
$$

12 for any Borel sets $B_{1}, B_{2}, \cdots, B_{m}$ of real numbers [22].

13 Liu [24] developed operation laws for uncertain variables so that the dis14 tribution of functions of independent uncertain variables can be achieved. Let

$15 \xi_{1}, \xi_{2}, \cdots, \xi_{n}$ be independent uncertain variables with regular uncertainty distri16 butions $\Phi_{1}, \Phi_{2}, \cdots, \Phi_{n}$, respectively. If the function $f\left(x_{1}, x_{2}, \cdots, x_{n}\right)$ is strictly 17 increasing with respect to $x_{1}, x_{2}, \cdots, x_{m}$, and strictly decreasing with respect 18 to $x_{m+1}, x_{m+2}, \cdots, x_{n}$, then, the uncertain variable $\xi=f\left(\xi_{1}, \xi_{2}, \cdots, \xi_{n}\right)$ has an 19 inverse uncertainty distribution

$$
\Psi^{-1}(\alpha)=f\left(\Phi_{1}^{-1}(\alpha), \Phi_{2}^{-1}(\alpha), \cdots, \Phi_{m}^{-1}(\alpha), \Phi_{m+1}^{-1}(\alpha), \cdots, \Phi_{n}^{-1}(\alpha)\right)
$$

20 3.2. Belief reliability as an uncertain measure

21 Belief reliability measures the degree to which we believe that a component or a system can perform its function as designed. In this subsection, we compare 
1 four mathematical theories commonly used to model belief degrees, probability

2 theory (subjective interpretation [7]), evidence theory [29], possibility theory [8]

3 and uncertainty theory [26], and choose among them the most appropriate one

4 as the mathematical foundation for belief reliability.

5 In practice, how to calculate the belief degree of the intersection of events

6 (more formally, the product event) is an important issue, since it is the basis

7 of system reliability calculations. Based on how the belief degree of the inter-

8 section of events is calculated, the four theories can be divided into two groups.

9 Probability theory and evidence theory comprise the first group, where the belief

10 degree of the intersection of events is calculated by the product of the individual 11 belief degrees (assuming independence among the individual events).

12 According to Liu [25], a premise of using the product operation to calculate 13 the belief degree of the intersection of events is that the estimated belief degree 14 for each individual event is close enough to the long-run cumulative frequency.

15 As shown in (8), however, belief reliability is a more conservative reliability mea16 sure than the probabilistic reliability. If we use probability theory or evidence 17 theory to model belief reliability, the conservatism in the component level will 18 be distorted by the product operation, which might lead to counter-intuitive re19 sults when calculating system belief reliability. To illustrate this point, consider 20 the following example.

21 Example 1. Consider a series system of 2000 components. Suppose for each 22 component, $m_{d}=9$ and $F_{a}=0$. It is easy to verify that both the component 23 and the system are unlikely to fail.

24 When using belief reliability as the reliability measure, we have to consider 25 the effect of epistemic uncertainty, by evaluating our state of knowledge. Sup26 pose for each component, we have $F_{e}=3$. Then, from (7), the belief reliability 27 of each component is $R_{B}=0.9987$. If we regard belief reliability as a probability 28 measure, the system belief reliability should be calculated by the product of the 29 component belief reliabilities:

$$
R_{B, S}=R_{B}^{200}=0.9987^{2000}=0.074
$$


1 Based on the evaluation result in (14), the system is highly unreliable, which 2 contradicts with our intuition.

3 Example 1 shows that to model belief reliability, we need a mathematical 4 theory whose operation law of product events can compensate for the conser5 vatism in the component-level belief reliability evaluation. Possibility theory 6 provides an alternative solution by assuming that the product belief degree is 7 the minimum one among all the individual events $[8,33]$. If we regard the com8 ponent belief reliabilities in Example 1 as a possibility measure, according to 9 [8], the system belief reliability is given by

$$
R_{B, S}=\bigwedge_{i=1}^{2000} R_{B, i}=0.9987,
$$

10 which avoids the counter-intuitive result in Example 1. However, regarding 11 belief reliability as a possibility measure introduces an issue: possibility measure 12 does not follow the duality axiom, which might lead to other counter-intuitive 13 results [24]. For instance, see Example 2.

14 Example 2. Assume that belief reliability $R_{B}$ is a possibility measure. A 15 possibility measure $\Pi$ has the following properties [8]:

- $\Pi(\Omega)=1$, where $\Omega$ is the universal set, and

- $\Pi(U \cup V)=\Pi(U) \vee \Pi(V)$, for any pair of disjoint sets $U$ and $V$.

Since "working" and "failure" are two disjoint sets and their union is the universal set, from the above axioms, it is easy to show that for a given component or a system, either the reliability $R_{B}=1$ or the unreliability $\overline{R_{B}}=1$ which will confuse the decision maker when applied in practice.

From Examples 1 and 2, we can see that to model belief reliability, we need a mathematical theory which can compensate the conservatism in the individual belief degree and satisfy the duality axiom. Compared to probability theory, uncertainty theory differs in the Product Axiom, where a minimum operator is used instead of the product operator, indicating that the uncertainty theory is 
1 capable to compensate for the extra dispersion induced by epistemic uncertainty.

2 Compared to possibility theory, uncertainty theory follows the Duality Axiom,

3 which prevents the counter-intuitive examples such as that in Example 2. Hence,

4 belief reliability is assumed to be an uncertain measure in this paper.

5 Definition 2 (Mathematical definition of belief reliability). Let the universal

6 set $\Gamma=\left\{\gamma_{1}, \gamma_{2}\right\}$, where $\gamma_{1}$ represents the working state of a system or compo-

7 nent, while $\gamma_{2}$ represents the failure state. Then, belief reliability $R_{B}$ is defined

8 as the uncertain measure of the event $\Lambda_{1}=\left\{\gamma_{1}\right\}$,

$$
R_{B}=\mathcal{M}\left\{\Lambda_{1}\right\}
$$

9 Remark 1. From the Duality Axiom, we can calculate the belief unreliability:

$$
\overline{R_{B}}=\mathcal{M}\left\{\Lambda_{2}\right\}=1-R_{B}
$$

10 which can also be seen from Figure 1, since the areas of failure region and safe 11 region sum up to 1.

\section{4. Minimal Cut Set Theorem}

13 In this section, we show how to calculate the belief reliability of a coherent 14 system by proving the Minimal Cut Set Theorem. Coherent system is the 15 most widely applied system model in reliability theory, which describes the 16 logic of binary monotone systems whose components are all relevant [3, 28].

17 Commonly encountered examples of coherent systems include series systems, 18 parallel systems, k-out-n:G systems, etc.

19 Let $\xi_{i}, 1 \leq i \leq n$ and $\xi$ denote the state of the $i$ th component and of the 20 system, respectively, where

$$
\xi_{i}=\left\{\begin{array}{l}
1, \text { if the } i \text { th component is working }, \\
0, \text { if the } i \text { th component fails. }
\end{array} \xi=\left\{\begin{array}{l}
1, \text { if the system is working } \\
0, \text { if the system fails. }
\end{array}\right.\right.
$$

21 The boolean variables $\xi$ and $\xi_{i}, 1 \leq i \leq n$ are referred to as state variables for 22 the system and the components, respectively. 
In coherent systems, $\xi$ is a function of $\xi_{i}, 1 \leq i \leq n$ :

$$
\xi=\phi\left(\mathbf{x}_{\xi}\right)=\phi\left(\xi_{1}, \xi_{2}, \cdots, \xi_{n}\right)
$$

2 where $\mathbf{x}_{\xi}=\left[\xi_{1}, \xi_{2}, \cdots, \xi_{n}\right]$ is the state vector of the components. The function $3 \phi(\cdot)$ in (19) is the structure function of the coherent system.

4 The state variables $\xi, \xi_{i}, 1 \leq i \leq n$ are all Boolean uncertain variables. Since

$5 \xi$ can be determined by $\xi_{1}, \xi_{2}, \cdots, \xi_{n}$ via the structure function, $\xi$ is a function

6 of uncertain variables. Hence its uncertainty distribution can be obtained via

7 the operation laws of uncertain variables [26]. Following the operation law for

8 Boolean uncertain variables, Liu [23] proved the Reliability Index Theorem for 9 coherent systems:

10 Theorem 1 (Reliability Index Theorem [23]). Assume that a system contains 11 uncertain elements $\xi_{1}, \xi_{2}, \cdots, \xi_{n}$ and has a structure function $\phi$. If $\xi_{1}, \xi_{2}, \cdots, \xi_{n}$ 12 are independent uncertain elements with reliability indices $a_{1}, a_{2}, \cdots, a_{n}$, respec13 tively, then, the system reliability index a is

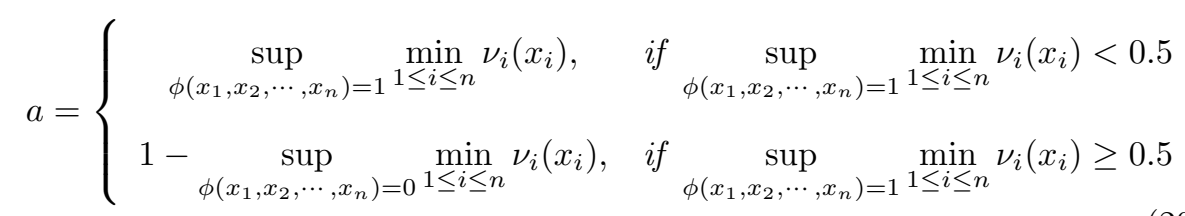

14 where $x_{i}, i=1,2, \cdots, n$ take value either 0 or 1 , and $\nu_{i}$ are defined by

$$
\nu_{i}\left(x_{i}\right)=\left\{\begin{array}{cc}
a_{i}, & \text { if } x_{i}=1 \\
1-a_{i}, & \text { if } x_{i}=0 .
\end{array}\right.
$$

15 The proof of Theorem 1 can be found in [23].

16 Directly applying Theorem 1 to calculate belief reliability of a coherent sys17 tem requires enumerating all possible combinations of $\xi_{i}$, which is tedious and 18 hard to apply in practice. In order to simplify the evaluation processes, we de19 velop a system belief reliability evaluation method for coherent systems based 20 on the concept of minimal cut sets.

21 Definition 3 (Minimal cut set). Suppose $\mathbf{x}=\left[x_{1}, x_{2}, \cdots, x_{n}\right]$ is the state vector 22 of a coherent system whose structure function is $\phi$. A vector $\mathbf{x}_{a}$ is called a 
1 minimal cut vector if $\phi\left(\mathbf{x}_{a}\right)=0$ and $\phi\left(\mathbf{x}_{b}\right)=1, \forall \mathbf{x}_{b}>\mathbf{x}_{a}$. By $\mathbf{x}_{b}>\mathbf{x}_{a}$, we

2 mean $\mathbf{x}_{b, i} \geq \mathbf{x}_{a, i}, 1 \leq i \leq n$ and there is at leat one $i, \mathbf{x}_{b, i}>x_{a, i}$.

3 Suppose $x_{C}$ is a minimum cut vector. Let $C\left(x_{C}\right)=\left\{i: x_{i}=0\right\}$. Then, $4 C\left(x_{C}\right)$ is referred to as a minimum cut set.

5 A minimal cut set is the smallest combination of components which will 6 cause the systems failure if they all fail. In [36], the authors used minimal cut 7 sets to reduce the computational costs in system belief reliability calculations.

8 However, their method requires a strict assumption that all the minimal cut

9 sets are independent. In this paper, we show that the restriction is unnecessary,

10 by proving the Minimal Cut Set Theorem, which only requires independence

11 among the components.

12 Theorem 2 (Minimal Cut Set Theorem). Consider a coherent system com13 prising $n$ independent components with belief reliabilities $R_{B, i}, i=1,2, \ldots, n$. If 14 the system contains $m$ minimal cut sets, $C_{1}, C_{2}, \ldots, C_{m}$, then, the system belief 15 reliability is

$$
R_{B, S}=\bigwedge_{1 \leq i \leq m} \bigvee_{j \in C_{i}} R_{B, j}
$$

Proof. Without loss of generality, let us assume that the $i$ th minimal cut set $C_{i}$ contains $n_{i}$ components. Let us also assume

$$
\begin{gathered}
R_{B, 11} \geq R_{B, 12} \geq \cdots R_{B, 1 j} \geq \cdots \geq R_{B, 1 n_{1}}, \\
R_{B, 21} \geq R_{B, 22} \geq \cdots R_{B, 2 j} \geq \cdots \geq R_{B, 2 n_{2}}, \\
\vdots \\
R_{B, m 1} \geq R_{B, m 2} \geq \cdots R_{B, m j} \geq \cdots \geq R_{B, m n_{m}},
\end{gathered}
$$

and

$$
R_{B, 11} \geq R_{B, 21} \geq \cdots R_{B, j 1} \geq \cdots \geq R_{B, m 1}
$$

16 where $R_{B, i j}$ denotes the belief reliability of the $j$ th component in the $i$ th minimal 17 cut set. In order to prove (22), we only have to prove

$$
R_{B, S}=R_{B, m 1}
$$


1 Equation (23) comes from the fact that $R_{B, 11}, R_{B, 21}, \cdots, R_{B, m 1}$ are the maxi-

2 mum component belief reliabilities for each minimal cut set, and $R_{B, m 1}$ is the 3 minimum among $R_{B, 11}, R_{B, 21}, \cdots, R_{B, m 1}$.

4 The proof breaks into two cases:

$5 \quad$ 1. If $R_{B, m 1}<0.5$ :

6 Since $\phi\left(x_{1}, x_{2}, \cdots, x_{n}\right)=1$ indicates that at least one component in each minimal cut set is working, it is easy to verify that

$$
\sup _{\phi\left(x_{1}, x_{2}, \cdots, x_{n}\right)=1} \min _{1 \leq i \leq n} \nu_{i}\left(x_{i}\right)=\min _{1 \leq i \leq m}\left\{\max _{\phi_{i}\left(x_{1}, x_{2}, \cdots, x_{n_{i}}\right)=1} \min _{1 \leq j \leq n_{i}} \nu\left(x_{i j}\right)\right\}
$$

8

9

10

11

12

where $\phi_{i}\left(x_{1}, x_{2}, \cdots, x_{n_{i}}\right)=\max _{1 \leq j \leq n_{i}} x_{i j}$.

Since $R_{B, m 1} \geq R_{B, m 2} \geq \cdots R_{B, m j} \geq \cdots \geq R_{B, m n_{m}}$, we have

$$
\begin{aligned}
\max _{\phi_{m}\left(x_{1}, x_{2}, \cdots, x_{n_{m}}\right)=1} \min _{1 \leq j \leq n_{m}} \nu\left(x_{i j}\right) & =\min \left(R_{B, m 1}, \min _{2 \leq j \leq n_{m}}\left(1-R_{B, m j}\right)\right) \\
& =R_{B, m 1} .
\end{aligned}
$$

For $1 \leq i \leq m-1$, if $R_{B, i 1} \geq 0.5$, from Lemma 1 in Appendix A, we have

$$
\max _{\phi_{i}\left(x_{1}, x_{2}, \cdots, x_{n_{i}}\right)=1} \min _{1 \leq j \leq n_{i}} \nu\left(x_{i j}\right) \geq 0.5>R_{B, m 1} ;
$$

if $R_{B, i 1}<0.5$, then, like $(25)$, we can prove that

$$
\max _{\phi_{i}\left(x_{1}, x_{2}, \cdots, x_{n_{i}}\right)=1} \min _{1 \leq j \leq n_{i}} \nu\left(x_{i j}\right)=R_{B, i 1} \geq R_{B, m 1} .
$$

Substituting (26) and (27) into (24), we have

$$
\sup _{\phi_{i}\left(x_{1}, x_{2}, \cdots, x_{n_{i}}\right)=1} \min _{1 \leq j \leq n_{i}} \nu\left(x_{i j}\right)=R_{B, m 1}<0.5 .
$$

Note that belief reliability is a reliability index. Then, from Theorem 1, $R_{B, S}=R_{B, m 1}$.

2. If $R_{B, m 1} \geq 0.5$ :

Since $R_{B, 11} \geq R_{B, 21} \geq \cdots R_{B, j 1} \geq \cdots \geq R_{B, m 1} \geq 0.5$, from Lemma 1 , we have

$$
\sup _{\phi\left(x_{1}, x_{2}, \cdots, x_{n_{i}}\right)=1} \min _{1 \leq j \leq n_{i}} \nu_{i}\left(x_{i}\right) \geq 0.5 .
$$



set, all the components fail, we have

$$
\begin{aligned}
\sup _{\phi\left(x_{1}, x_{2}, \cdots, x_{n}\right)=0} \min _{1 \leq i \leq n} \nu\left(x_{i}\right) & =\max _{1 \leq i \leq m} \min _{1 \leq j \leq n_{i}}\left(1-R_{B, i j}\right) \\
& =\max _{1 \leq i \leq m}\left(1-R_{B, i 1}\right)=1-R_{B, m 1} .
\end{aligned}
$$

Then, from Theorem 1,

$$
R_{B, S}=1-\sup _{\phi\left(x_{1}, x_{2}, \cdots, x_{n}\right)=0} \min _{1 \leq i \leq n} \nu\left(x_{i}\right)=R_{B, m 1}
$$

4

5 Example 3 (Belief reliability of a series system). Consider a series system com6 prising $n$ independent components with belief reliabilities $R_{B, i}, i=1,2, \ldots, n$.

7 It is easy to show that the system has $n$ minimal cut sets, $C_{1}=\{1\}, C_{2}=$ $8\{2\}, \ldots, C_{n}=\{n\}$. Therefore, from Theorem 2, the belief reliability of the 9 system is

$$
R_{B, S}=\bigwedge_{1 \leq i \leq n} R_{B, i} .
$$

10 Reference [23] also calculates the belief reliability of a series system using 11 the Reliability Index Theorem. The result in (32) is the same as that from using 12 Theorem 1 ([23]). However, using Theorem 1 requires $n \cdot 2^{n}$ comparisons, while 13 using Theorem 2 requires only $n$ comparisons. Therefore, the computational 14 costs can be greatly reduced by using the Minimal Cut Set Theorem.

15 Example 4 (Belief reliability of a parallel system). Consider a paralell sys16 tem comprising $n$ independent components with belief reliabilities $R_{B, i}, i=$ $171,2, \ldots, n$. It is easy to show that the system has one minimal cut set, $C_{1}=$ $18\{1,2, \ldots, n\}$. Therefore, from Theorem 2 , the system belief reliability is

$$
R_{B, S}=\bigvee_{1 \leq i \leq n} R_{B, i}
$$

19 Reference [23] also calculates the belief reliability of a parallel system using

20 the Reliability Index Theorem. The result in (33) is the same as that from using 21 Theorem 1 ([23]). However, using Theorem 1 requires $n \cdot 2^{n}$ comparisons, while 
1 using Theorem 2 requires only $n$ comparisons. Therefore, the computational

2 costs can be greatly reduced by using the Minimal Cut Set Theorem.

3 Example 5 (Belief reliability of a k-out-n:G system). Consider a k-out-n:G 4 system comprising $n$ independent components with belief reliabilities $R_{B, i}, i=$

$51,2, \ldots, n$. It is easy to show that the system has $C_{n}^{(k+1)}$ minimal cut sets.

6 Each minimal cut set contains $k+1$ components arbitrary chosen from the $n$

7 components. Therefore, from Theorem 2, the belief reliability of the system is

$$
R_{B, S}=R_{B, k}
$$

8 Reference [23] also calculates the belief reliability of a k-out-n:G system using

9 the Reliability Index Theorem. The result in (34) is the same as that from using

10 Theorem 1 ([23]). However, using Theorem 1 requires $n \cdot 2^{n}$ comparisons, while

11 using Theorem 2 requires only $n$ comparisons. Therefore, the computational

12 costs can be greatly reduced by using the Minimal Cut Set Theorem.

\section{5. Fault tree analysis using belief reliability}

14 In this section, we show how to calculate system belief reliability based on 15 fault tree models. For this, we first show that Theorem 2 also applies to cut 16 sets. A vector $\mathbf{x}_{C S}$ is a cut vector if $\phi\left(\mathbf{x}_{C S}\right)=0$. Then, $C S=\left\{i: x_{C S, i}=0\right\}$ is 17 defined as a cut set. All minimal cut sets are cut sets; whereas, a cut set might 18 be necessarily be a minimal cut set since it might contain redundant elements. 19 If a cut set $C S$ comprises of all the elements of a minimal cut set $C$ and some 20 redundant elements, $C$ is said to be contained in $C S$.

21 Theorem 3 (Cut Set Theorem). Suppose that a coherent system has $m$ mini22 mal cut set $C S_{1}, C S_{2}, \cdots, C S_{m}$ and $(l-m)$ cut sets $C S_{m+1}, C S_{m+2}, \cdots, C S_{l}$ 23 that contain some minimal cut sets. Then, the system belief reliability can be 24 calculated by

$$
R_{B, S}=\bigwedge_{1 \leq i \leq l} \bigvee_{j \in C S_{i}} R_{B, j}
$$


1 Proof. Let

$$
R_{B, M C S}=\bigwedge_{1 \leq i \leq m} \bigvee_{j \in C S_{i}} R_{B, j}
$$

2 Without loss of generality, let us assume that $C S_{m+1}$ contains $C S_{1}$ and belief

3 reliabilities of the redundant components are $R_{B, R, 1} \geq R_{B, R, 2} \geq R_{B, R, n_{R}}$. Let

$4 R_{B, 1}$ denote the highest belief reliability among the components in $C S_{1}$.

5 If $R_{B, R, 1} \leq R_{B, 1}$, immediately we have

$$
R_{B, M C S}=\bigwedge_{1 \leq i \leq m+1} \bigvee_{j \in C S_{i}} R_{B, j}
$$

6

7 If $R_{B, R, 1}>R_{B, 1},(37)$ also holds since

$$
\bigvee_{j \in C S_{m+1}} R_{B, j}=R_{B, R, 1}>R_{B, 1}
$$

8

9 Similarly, we can prove that

$$
\bigwedge_{1 \leq i \leq l} \bigvee_{j \in C S_{i}} R_{B, j}=R_{B, M C S}
$$

10

11 From Theorem 2, $R_{B, M C S}=R_{B, S}$. Hence, the theorem is proved.

12 The cut sets required in (35) can be enumerated from the fault tree mod-

13 el using the MOCUS algorithm [11]. System belief reliability can, then, be

14 calculated by the following algorithm:

15 An engineering system, the left leading edge flap (LLEF) control subsystem

16 of the F-18 air fighters [9], is used to demonstrate the developed system belief

17 reliability analysis method. The schematic of the system is given in Fig. 2,

18 where FCC represents flight control computer, $\mathrm{CH}$ represents channel, HSA

19 represents hydraulic servo- actuator, LLEF represents left leading edge flap and

20 RLEF represents right leading edge flap [9].

21 The failure behavior of the system can be described by a fault tree, as shown

22 in Fig. 3 [9]. In Fig. 3, the basic events $1-9$ represent the failure of HSA-A, 


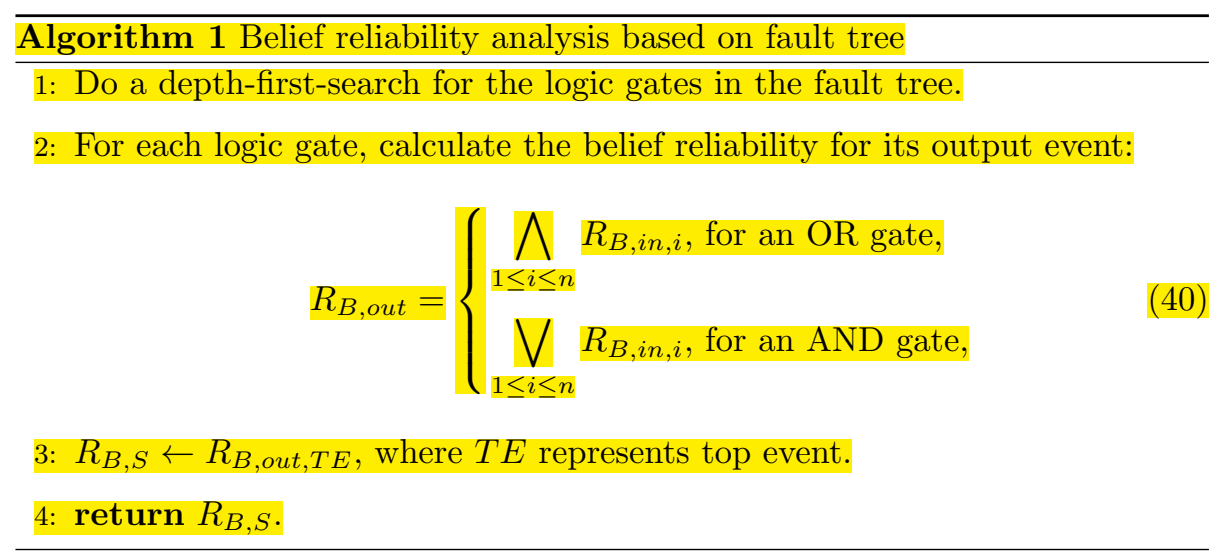

1 left asymmetry control unit, LLEF, CH1, CH2, CH3, CH4, FCC-A and FCC-B, 2 respectively.

3 The belief reliability of the components can be evaluated using the proce-

4 dures in [37]. Suppose the component belief reliabilities are $R_{B, 1}=0.9688, R_{B, 2}=$ $50.9200, R_{B, 3}=0.9500, R_{B, 4}=0.9000, R_{B, 5}=0.8000, R_{B, 6}=0.8800, R_{B, 7}=$ $60.9600, R_{B, 8}=0.9700, R_{B, 9}=0.9500$, respectively. From Algorithm 1, the be-

7 lief reliability of the system is

$$
\begin{aligned}
R_{B, S}= & R_{B, 1} \wedge R_{B, 2} \wedge R_{B, 3} \wedge \\
& \left(R_{B, 5} \wedge R_{B, 8}\right) \vee\left(R_{B, 6} \wedge R_{B, 9}\right) \wedge \\
& \left(R_{B, 4} \vee R_{B, 5} \vee R_{B, 6} \vee R_{B, 7}\right)
\end{aligned}
$$

8 Then, from (41), the belief reliability of the LLEF control system is $R_{B, S}=$ $9 R_{B, 6}=0.8800$.

10 The structure function-based method is also used to evaluate the system 11 belief reliability. To do this, all the possible combinations of the system states 12 need to be enumerated, which, in this case, are $2^{9}=512$ states. Then, the 13 system belief reliability is calculated based on (20). The calculated system belief 14 reliability is $R_{B, S}=0.8800$, which is the same as the one from Algorithm 1 .

15 According to (20), the structure function-based method requires $n \times 2^{n}=4608$ 16 comparisons, where $n$ is the number of components. Algorithm 1, however, 17 requires only 10 comparisons according to (41). The results demonstrate that 
1 using the developed methods can help to improve the computational efficiency

2 of system belief reliability analysis.

\section{6. Conclusion}

4 In this paper, belief reliability was defined as an uncertain measure in uncer-

5 tainty theory, due to the explicit representation of epistemic uncertainty. The

6 Minimal Cut Set Theorem was proved, which shows how to calculate the be-

7 lief reliability for coherent systems based on minimal cut sets. A system belief

8 reliability analysis method is, then, developed based on fault tree models and

9 applied on some numerical case studies. A comparison to the existing structure

10 function-based method shows that the developed methods reduces the compu-

11 tational costs in system belief reliability analysis.

12 In this paper, we only consider binary systems. Many practical systems, 13 however, are multi-state. In the future, the belief reliability evaluation method

14 will be extended to multi-state system models. Also, the belief reliability con15 sidered in this paper is independent of time. How to model the time-dependent

16 belief reliability is another future research direction.

\section{Acknowledgment}

18 This work has been performed within the initiative of the Center for Re19 silience and Safety of Critical Infrastructures (CRESCI, http://cresci.cn). The 20 research of Prof. Rui Kang is supported by National Natural Science Founda21 tion of China (NSFC) [grant number 61573043]. The research of Dr. Meilin

22 Wen is supported by National Natural Science Foundation of China (NSFC) 23 [grant number 71671009].

\section{References}

25 [1] Aven, T., Krohn, B., 2014. A new perspective on how to understand, assess and manage risk and the unforeseen. Reliability Engineering and System Safety 121, 1-10. 
1 [2] Aven, T., Zio, E., 2013. Model output uncertainty in risk assessment.

International Journal of Performability Engineering 9.

[3] Bayramoglu, I., Ozkut, M., 2015. The reliability of coherent systems subjected to marshall-olkin type shocks. IEEE Transactions on Reliability 64, 435-443. doi:10.1109/TR. 2014.2338252.

[4] Bjerga, T., Aven, T., Zio, E., 2014. An illustration of the use of an approach for treating model uncertainties in risk assessment. Reliability Engineering and System Safety 125, 46-53. doi:10.1016/j.ress.2014.01.014.

[5] Chamberlain, S., Chookah, M., Modarres, M., 2009. Development of a probabilistic mechanistic model for reliability assessment of gas cylinders in compressed natural gas vehicles. Proceedings of the Institution of Mechanical Engineers Part 0: Journal of Risk and Reliability 223, 289-299. doi: $\{10.1243 / 1748006 X$ JRR231\}.

[6] Collins, J., Busby, H., Staab, G., 2009. Mechanical design of machine elements and machines. Wiley.

[7] De Finetti, B., 1972. Probability, induction, and statistics .

[8] Dubois, D., Prade, H., 1988. Possibility theory: An approach to the computerized processing of information.

[9] Dugan, S.A.D.J., 1993. A quantitative analysis of the f18 flight control system, in: 9th Computing in Aerospace Conference San Diego,CA,U.S.A. Available from: http://dx.doi.org/10.2514/6.1993-4574.

[10] Fan, M., Zeng, Z., Kang, R., 2015. An approach to measure reliability based on belief reliability. Journal of Systems Engineering and Electronics $11,2648-2653$.

[11] Fussell, J.B., Vesely, W.E., 1972. A new methodology for obtaining cut sets for fault trees. Trans. Amer. Nuc. Soc. 15, 262. 
1 [12] Gao, R., Yao, K., 2016. Importance index of components in uncertain random systems. Knowledge-based Systems doi:10.1016/j.knosys. 2016. 07.006. accepted for publication.

[13] Gao, Y., Yang, L., Li, S., Kar, S., 2015. On distribution function of the diameter in uncertain graph. Information Sciences 296, 61-74. doi:10 . 1016/j.ins.2014.10.048.

[14] Han, S., Peng, Z., Wang, S., 2014. The maximum flow problem of uncertain network. Information Sciences 265, 167-175. doi:10.1016/j . ins . 2013.11. 029.

[15] Huang, M., Ren, L., Lee, L.H., Wang, X., Kuang, H., Shi, H., 2016. Model and algorithm for 4plrp with uncertain delivery time. Information Sciences 330, 211-225. doi:10.1016/j.ins.2015.10.030.

[16] Ji, X., Zhou, J., 2015. Option pricing for an uncertain stock model with jumps. Soft Computing 19, 3323-3329.

[17] Jiang, X., Zeng, Z., Kang, R., Chen, Y., 2015. A naive bayes based method for evaluating the effects of electronic product reliability simulation experiments. Electronic Science and Technology 1, 49-54.

[18] Kang, R., Zhang, Q., Zeng, Z., Zio, E., Li, X., 2016. Measuring reliability under epistemic uncertainty: A review on non-probabilistic reliability metrics. Chineses Jounal of Aeronautics 29, 571-579.

[19] Ke, H., Yao, K., 2016. Block replacement policy with uncertain lifetimes. Reliability Engineering and System Safety 148, 119-124. doi:10.1016/j . ress. 2015.12 .008$.

[20] Kiureghian, A., Ditlevsen, O., 2009. Aleatory or epistemic? does it matter? Structural Safety 31, 105-112.

[21] Liu, B., 2007. Uncertainty theory (Second Edition). Springer-Verlag, Berlin. 
1 [22] Liu, B., 2009. Some research problems in uncertainty theory. Journal of 2 Uncertain Systems 3, 3-10.

3 [23] Liu, B., 2010a. Uncertain risk analysis and uncertain reliability analysis. 4 Journal of Uncertain Systems 4, 163-170.

5 [24] Liu, B., 2010b. Uncertainty theory: A branch of mathematics for modeling 6 human uncertainty. Springer-Verlag, Berlin.

7 [25] Liu, B., 2012. Why is there a need for uncertainty theory. Journal of 8 Uncertain Systems 1, 3-12.

9 [26] Liu, B., 2015. Uncertainty theory, Fifth Edition. Springer.

10 [27] Liu, W., 2013. Reliability analysis of redundant system with uncertain lifetimes. Information: An International Interdisciplinary Journal 16, 881888.

[28] Navarro, J., Pellerey, F., Di Crescenzo, A., 2015. Orderings of coherent systems with randomized dependent components. European Journal of Operational Research 240,127-139. doi:10.1016/j.ejor.2014.06.020.

[29] Shafer, G., 1976. A mathematical theory of evidence. volume 1. Princeton university press Princeton.

[30] Wen, M.L., Kang, R., 2016. Reliability analysis in uncertain random system. Fuzzy Optimization and Decision Making Accepted for publication, http://orsc.edu.cn/online/120419.pdf.

[31] Yang, G., 2007. Life cycle reliability engineering. John Wiley and Sons, Hoboken, N.J.

[32] Yang, L., Liu, P., Li, S., Gao, Y., Ralescu, D.A., 2015. Reduction methods of type-2 uncertain variables and their applications to solid transportation problem. Information Sciences 291, 204-237. doi:10.1016/j.ins. 2014. 08.044 . 
1 [33] Zadeh, L.A., 1978. Fuzzy sets as a basis for a theory of possibility. Fuzzy

25 Lemma 1. Consider a coherent system comprising $n$ independent components 26 Sets and Systems 1, 3-28.

[34] Zeng, Z., Kang, R., Chen, Y., 2016a. Using pof models to predict system reliability considering failure collaboration. Chinese Journal of Aeronautics Accepted for publication.

[35] Zeng, Z., Kang, R., Wen, M., Chen, Y., 2015. Measuring reliability during product development considering aleatory and epistemic uncertainty, in: Reliability and Maintainability Symposium (RAMS), pp. 1-6.

[36] Zeng, Z., Wen, M., Kang, R., 2013. Belief reliability: a new metrics for products' reliability. Fuzzy Optimization and Decision Making 12, 15-27.

[37] Zeng, Z., Wen, M., Kang, R., Zio, E., 2016b. A new metric of reliability: Belief reliability. Chinese Journal of Aeronautics Under submission.

[38] Zhang, B., Peng, J., Li, S., 2015. Uncertain programming models for portfolio selection with uncertain returns. International Journal of Systems Science 46, 2510-2519.

[39] Zhang, C., Guo, C., 2013. Some new results on uncertain age replacement policy. Industrial Engineering and Management Systems 12, 41-45.

[40] Zhang, C., Guo, C., 2014. Uncertain block replacement policy with no replacement at failure. Journal of Intelligent and Fuzzy Systems 27, 19911997.

[41] Zio, E., Apostolakis, G., 1996. Two methods for the structured assessment of model uncertainty by experts in performance assessments of radioactive waste repositories. Reliability Engineering and System Safety 54, 225-241.

\section{Appendix A. Lemma 1 and its proof} with belief reliabilities $R_{B, i}, i=1,2, \cdots, n$, where $R_{B, 1} \geq R_{B, 2} \geq \cdots \geq R_{B, n}$. 
1 If the structure function of the system $\phi$ is:

$$
\phi\left(x_{1}, x_{2}, \cdots, x_{n}\right)=\max _{1 \leq i \leq n} x_{i},
$$

2 and there is at least one $R_{B, i}$ such that $R_{B, i} \geq 0.5$, then we have

$$
\sup _{\phi\left(x_{1}, x_{2}, \cdots, x_{n}\right)=1} \min _{1 \leq i \leq n} \nu_{i}\left(x_{i}\right) \geq 0.5 .
$$

3 Proof. The proof breaks into two cases:

$4 \quad 1$. If $R_{B, n} \geq 0.5$ :

5

Since $\phi(1,1, \cdots, 1)=1$, we have

$$
\sup _{\phi\left(x_{1}, x_{2}, \cdots, x_{n}\right)=1} \min _{1 \leq i \leq n} \nu_{i}\left(x_{i}\right) \geq \min _{1 \leq i \leq n} \nu_{i}(1)=R_{B, n} \geq 0.5 .
$$

$6 \quad 2$. If $R_{n}<0.5$ :

7 Without loss of generality, we assume that there exists a $k, k \in[1, n-1]$,

8 such that $R_{B, k} \geq 0.5$. Since $R_{n}<0.5$, there exists a $j \in(k, n)$, where

$9 \quad R_{j} \geq 0.5 \geq R_{j+1}$. It is easy to verify that $\phi\left(x_{1}, x_{2}, \cdots, x_{n}\right)=1$ where

$$
x_{i}=\left\{\begin{array}{l}
1, i=1,2, \cdots, j \\
0, i=j, j+1, \cdots, n .
\end{array}\right.
$$

10 Besides, for the $x_{i}, 1 \leq i \leq n$ in (A.4), we have

$$
\min _{1 \leq i \leq n} \nu_{i}\left(x_{i}\right)=\min \left(\min _{1 \leq i \leq j} \nu_{j}(1), \min _{j+1 \leq i \leq n} \nu_{j}(0)\right) \geq 0.5 .
$$

11 Therefore,

$$
\sup _{\phi\left(x_{1}, x_{2}, \cdots, x_{n}\right)=1} \min _{1 \leq i \leq n} \nu_{i}\left(x_{i}\right) \geq 0.5
$$




\section{List of Figures}

1 Epistemic uncertainty effect on the aleatory distribution of the performance margin (Adapted from [37]) . . . . . . . . . 26

2 Schematic diagram of the F-18 LLEF [9] . . . . . . . . . . 27

3 The fault tree of the F-18 LLEF $[9] \ldots \ldots \ldots \ldots$ 


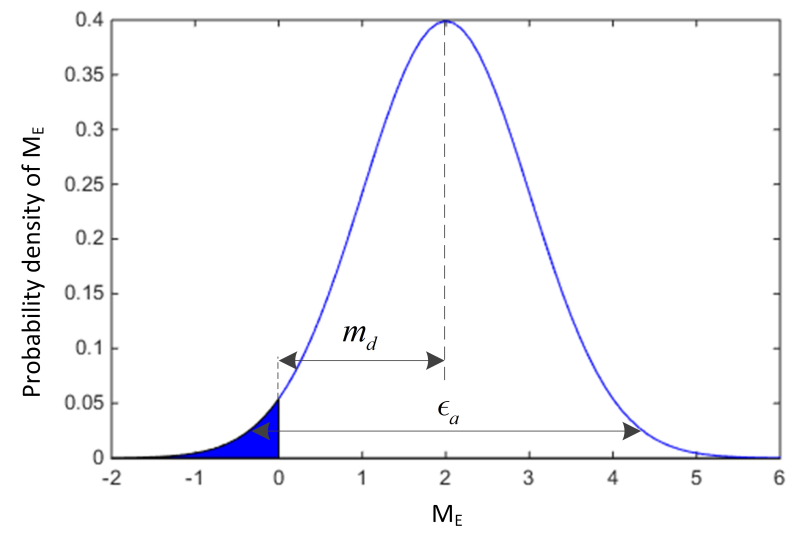

(a) Aleatory distribution of the performance margin

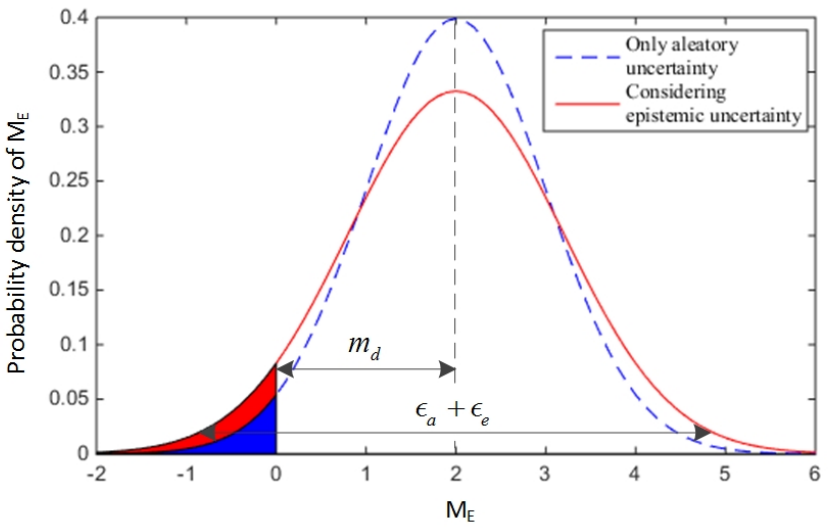

(b) Effect of epistemic uncertainty

Figure 1: Epistemic uncertainty effect on the aleatory distribution of the performance margin (Adapted from [37]) 


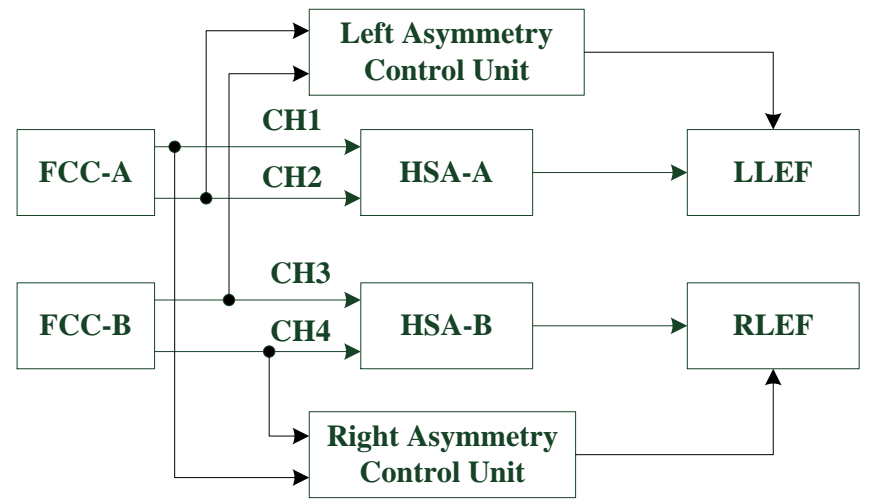

Figure 2: Schematic diagram of the F-18 LLEF [9]

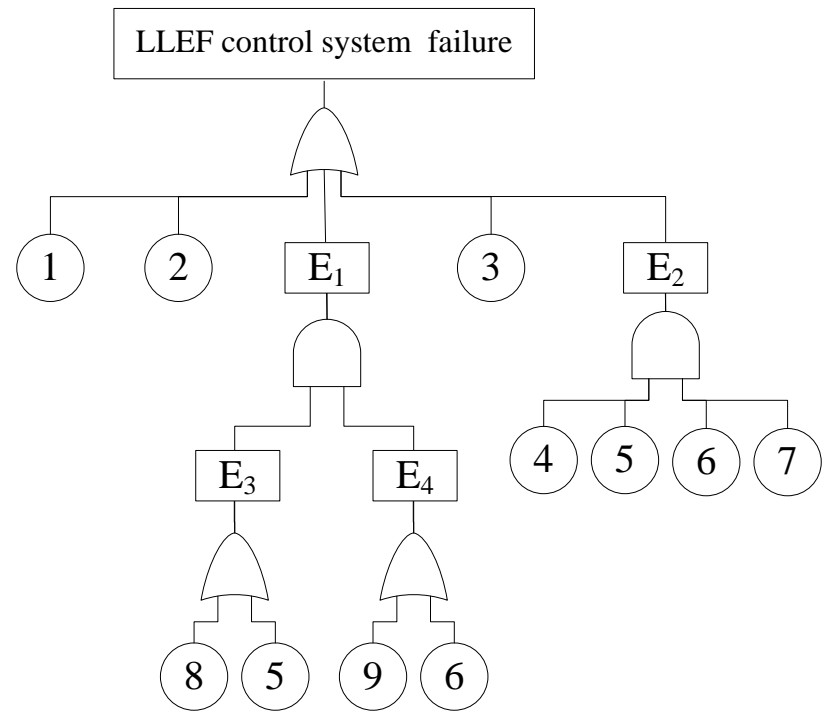

Figure 3: The fault tree of the F-18 LLEF [9] 